\title{
Begleitetes Sterben in Deutschland und der Schweiz: Ein aktueller Blick über die Grenzen
}

Karl-Ludwig Kunz

\section{Sterbetourismus}

Die organisierte Freitodbegleitung ist in der Schweiz eine etablierte Praxis. Die grösste Sterbehilfeorganisation EXIT hat 53`000 Mitglieder und führt jährlich effektiv etwa 200 Freitodbegleitungen durch. ${ }^{1}$ Die kleinere, in ihrer Praxis umstrittenere Organisation DIGNITAS betreibt Suizidhilfe auch gegenüber Personen mit Wohnsitz im Ausland. Sie hat knapp 6000 Mitglieder, davon 2,722 Deutsche, wobei 1,737 ihrer deutschen Sektion angehören. ${ }^{2}$ Durch DIGNITAS wurden bis Ende 2010 total 1138 Freitode begleitet, wobei die Suizidenten zu 87,61\% ihr Domizil im Ausland und zu 62,18\% in Deutschland hatten. Die meisten Freitodbegleitungen jährlich führte DIGNITAS 2006 mit 195 Fällen durch; 2010 waren es noch 97 Fälle. $^{3}$

Suizidhilfe wird in der Schweiz zur Sicherung eines menschenwürdigen Sterbens geleistet, und zwar für dies verantwortlich Wünschende mit ärztlich diagnostizierter hoffnungsloser Prognose, unerträglichen Beschwerden oder unzumutbarer Behinderung ${ }^{4}$. Der Einsatz des dem Betäubungsmittelgesetz unterfallenden ${ }^{5}$ tödlich wirkenden Natrium-Pentobarbital $(\mathrm{NaP})$ setzt ein ärztliches Rezept voraus. Über der Praxis der Rezeptabgabe liegt ein kaum durchdringlicher Grauschleier. Zu vermuten ist, dass entsprechende Rezepte insbesondere durch sog. „Vertrauensärzte“ der Sterbehilfeorganisationen sogar für Depressive grosszügig ausgestellt werden. ${ }^{6}$ Damit werden die Ärzte zum weitgehend unkontrollierten Pförtner zu einem schmerzfreien zuverlässigen Freitod.

Trotzdem hat DIGNITAS wiederholt vergeblich versucht, die Rezeptpflicht zu umgehen. So reichte sie mit dem Ziel, auch bei Gesunden Sterbehilfe leisten zu können, ${ }^{7}$ ein Gesuch bei der Bundesregierung um eine Sonderbewilligung ein, das Barbiturat ohne ärztliche Verschreibung zu beziehen und treuhänderisch für Sterbewillige verwenden zu können. ${ }^{8}$ Ferner hat ein sterbewilliges Mitglied dieser Organisation den Rechtsweg mit dem Ziel eines rezeptfreien Bezugs des Barbiturats über DIGNITAS beschritten. Das schweizerische Bundesgericht hat dies mit der Begründung abgelehnt, dass es im Rahmen der organisierten Suizidbeihilfe keinen verfassungsrechtlichen Anspruch auf Abgabe einer tödlichen Dosis NaP ohne Rezept gebe. ${ }^{9}$ Eine daraufhin eingereichte Beschwerde beim Europäischen Gerichtshof für Menschenrechte wurde ebenfalls abgelehnt. ${ }^{10} \mathrm{Nach}$ dem Anfang 2008 der Zürcher Kantonsarzt die seit bald zehn Jahren übliche Praxis, einem Sterbewilligen nach einem Gespräch mit einem Arzt ohne weiteres das erforderliche Rezept auszustellen, als nicht mehr der Anforderung an die ärztliche Sorgfaltspflicht genügend bezeichnet hatte, hat DIGNITAS vereinzelt das rezeptfrei erhältliche Heliumgas unter einer Maske, die sich der Suizidwillige über Mund und Nase zu ziehen hat, als Tötungsmittel eingesetzt. ${ }^{11}$ Nachdem DIGNITAS auf Drängen der Nachbarschaft angemietete Wohnungen gekündigt wurden, hat sie ihre Tätigkeit in Autos oder Wohnwagen auf öffentlichen Parkplätzen („mobile Sterbezimmer“) und Hotels verlagert. ${ }^{12}$ Auch wurde durch DIGNITAS-Mitarbeiter offenbar heimlich die Asche von Suizidenten im Zürich-See entsorgt. ${ }^{13}$ Verschiedentlich haben DIGNITAS und EXIT Suisse romande ver- sucht, sich freien Zugang zu Altersheimen und Krankenhäusern zu verschaffen. Von EXIT wurden NaP-Reservedosen illegal gelagert. Insgesamt ist eine zunehmende Werbetätigkeit von Sterbehilfeorganisationen mittels Inseraten und Berichten im In- und Ausland, Auftritten an Gemeindeanlässen und Radiospots zu beobachten. ${ }^{14}$

DIGNITAS hat 2005 die deutsche Sektion DIGNITATE gegründet, deren Mitglieder volles Anrecht auf Suizidhilfe in der Schweiz haben. In Deutschland wird die Freitodbegleitung jedoch nicht praktiziert. Als Gründe gibt DIGNITATE das Fehlen einer gefestigten Gerichtspraxis zur Suizidbegleitung in Deutschland an. Hinzu komme, dass Natrium-Pentobarbital (NaP) in Deutschland in der erforderlichen Dosis nicht ärztlich verschrieben werden dürfe. ${ }^{15}$ Dazu heisst es im jüngsten Jahresbericht der deutschen Sektion: „Aufgrund der in Deutschland herrschenden Rechtslage ist es zurzeit noch immer nicht möglich, den Mitgliedern des Vereins auf deutschem Boden eine risiko- und schmerzfreie Freitod-Begleitung anzubieten. Nach wie vor müssen deshalb Deutsche ihrer persönlichen Freiheit zuliebe mit den Füssen abstimmen und sich in ein Land begeben, welches die Verpflichtung, die persönliche Freiheit der Menschen zu schützen, nicht nur als Lippenbekenntnis versteht. “16

\section{Rechtslage in Deutschland und in der Schweiz}

Was unterscheidet die Rechtslage zum begleiteten Suizid Deutschlands von derjenigen in der Schweiz? ${ }^{17}$ Genau betrachtet Details, die sich als folgenreich erweisen. In beiden Ländern ist die Fremdtötung durch aktive Lebensverkürzung grundsätzlich strafbar, und zwar sogar dann, wenn sie auf Grund eines ausdrücklichen und ernstlichen Verlangens des Getöteten erfolgt ist ( $\$ 216$ d StGB, Art. 114 ch $\mathrm{StGB}$ ). Eine Fremdtötung liegt vor, wenn die Tatherrschaft über das zum Tode führende Geschehen nicht beim Sterbewilligen, sondern bei dem Dritten liegt. Wer dem Sterbewilligen etwa eine tödliche Injektion oder Infusion beibringt, tötet einen anderen und macht sich wegen Fremdtötung hier wie dort strafbar. Die Strafwürdigkeit dieser „direkte aktive Sterbehilfe“ genannten Fallkonstellation ist mit Ausnahme des Extremfalls der Beseitigung unerträglicher, auch palliativmedizinisch nicht linderbarer Leiden von Sterbewilligen, die unfähig sind, sich selbst durch den Tod zu erlösen, unumstritten. ${ }^{18}$ Die palliative ärztliche Anwendung schmerzlindernder Mittel in der Endphase eines ansonsten mit unerträglichen Schmerzen verbundenen Sterbeprozesses stellt formal betrachtet ebenfalls eine Fremdtötung dar, wenn sich darin das Risiko einer Lebensverkürzung verwirklicht („indirekte aktive Sterbehilfe“). Indessen gilt dieses Risiko angesichts des ärztlichen Gebots der Leidensminderung als erlaubt. ${ }^{19}$ $\mathrm{Da}$ das erlaubte Risiko beliebig hoch und nahezu Gewissheit erreichen kann, wird die zulässige Leidensverminderung mit dem Risiko der Lebensverkürzung von der unzulässigen leidensmindernden Lebensverkürzung praktisch kaum mehr unterscheidbar. Dies führt in der Praxis der Schweiz zu einer rechtlichen Grauzone des extensiven Einsatzes palliativer Techniken. ${ }^{20}$ Erst recht erlaubt ist die Hilfe im Sterben durch blosse Schmerzlinderung ohne lebensverkürzendes Risiko. ${ }^{21}$ Als ebenfalls rechtmässig wird die Konstellation 
des ärztlichen Behandlungsabbruchs angesehen, wenn dies dem tatsächlichen oder mutmaßlichen Patientenwillen entspricht und dazu dient, einem ohne Behandlung zum Tode führenden Krankheitsprozess seinen Lauf zu lassen. ${ }^{22}$ Damit wird dem mit dem Segen der Reanimationstechnik verbundenen Fluch einer nur noch künstlichen apparativen Lebenserhaltung abgeholfen. Verbreitet, in Deutschland und künftig auch in der Schweiz (siehe den folgenden Beitrag von Eicker / Fisch) gesetzlich geregelt ${ }^{23}$ ist die sog. Patientenverfügung, mittels derer schriftlich für den Fall der Entscheidungsunfähigkeit für Arzt oder Betreuer Handlungs- oder Unterlassungsanweisungen für Heilbehandlungen oder ärztliche Eingriffe gegeben werden.

Auch wo keine Fremdtötung vorliegt, sondern die Tatherrschaft bei dem sich tötenden Sterbewilligen liegt und ein Dritter dazu Hilfe leistet, ist die Rechtslage in beiden Nationen ähnlich. Da die frei verantwortliche Selbsttötung nicht strafbar ist, gilt für den Versuch derselben und die Anstiftung dazu oder die Hilfe dafür dasselbe. Nur ausnahmsweise ist als Suizidhelfer nach schweizerischem Recht (Art. 115 ch-StGB) strafbar, „wer aus selbstsüchtigen Beweggründen jemand zum Selbstmorde verleitet oder ihm dazu Hilfe leistet“. Eine selbstsüchtige Betätigung konnte der organisierten Suizidhilfe bislang nicht nachgewiesen werden. Immerhin wurde DIGNITAS 2007 von Amtes wegen als nach kaufmännischer Art geführtes Gewerbe ins Handelsregister eingetragen und damit einer gesetzlichen Buchführungspflicht unterworfen. ${ }^{24}$

Die ausnahmsweise Strafbarkeit der selbstsüchtigen Suizidbeteiligung wird als abschliessend verstanden. Damit wird Überlegungen eine Absage erteilt, die Suizidbeihilfe als strafbares Unterlassen einer gebotenen Betätigung zur Lebenserhaltung zu verstehen. In Deutschland, wo eine solche exzeptionelle Strafvorschrift für eine speziell motivierte Suizidbeteiligung fehlt, fehlt es konsequenterweise auch an der mit ihr verbundenen Sperrwirkung der Exklusivität. Deshalb ist in Deutschland die Möglichkeit der Umdeutung der Suizidbeihilfe in eine strafbare Tötung durch Unterlassen ( $\mathbb{S} \mathbb{S} 211 \mathrm{ff} ., 13 \mathrm{~d}$-StGB) oder in eine Unterlassene Hilfeleistung ( $\$ 323 \mathrm{c} d-S t G B)$ nicht grundsätzlich versperrt.

Die deutsche Rechtslage ist insoweit umstritten. Nach herrschender Lehre begründet weder die Nichthinderung des Suizids noch der Verzicht auf Rettungsmassnahmen eine Strafbarkeit, wenn von einem bis zum tödlichen Ende durchgehaltenen Suizidwillen ausgegangen werden kann. ${ }^{25}$ Dem folgend, formuliert der Alternativentwurf Sterbehilfe in $\ 215$ Abs. 1 wie folgt: „Wer es unterlässt, die Selbsttötung eines anderen zu hindern, handelt nicht rechtswidrig, wenn die Selbsttötung auf einer freiverantwortlichen, ausdrücklich erklärten oder aus den Umständen erkennbaren ernstlichen Entscheidung beruht." Demgegenüber vertritt die Rechtsprechung des Bundesgerichtshofs unter Billigung durch eine Mindermeinung des Schrifttums beim tatenlosen Geschehenlassen einer Selbsttötung durch einen zur Erfolgsabwendung Verpflichteten eine Rettungspflicht und damit eine Haftung wegen Tötung durch Unterlassen ( $\mathbb{S} 211 \mathrm{ff}$., 13 d-StGB). Als Verpflichtungsgrund wird neben der Beschützerfunktion des Angehörigen oder Arztes bereits die vorangegangene aktive Beihilfe zum Suizid durch einen beliebigen Anderen angesehen, obwohl diese aktive Unterstützung erlaubt ist, was zu einem Widerspruch mit der grundsätzlichen Wertentscheidung des Gesetzes für die Straflosigkeit der aktiven Suizidbeteiligung führt. Bei Vorliegen einer solchen Garantenstellung hat der BGH dies zunächst bei jedweder Nichthinderung eines Suizids vertreten. ${ }^{26}$ Später wurde ein rettungspflichtbegründender Unglücksfall ab dem Zeitpunkt, in dem das ursprünglich vom freien Willen getragene suizidale Gesche- hen vom Suizidenten nicht mehr beherrschbar ist, angenommen. ${ }^{27}$ Dies wurde mit verschiedenen Einschränkungen versehen, dass der Garant das Suizidgeschehen beherrscht ${ }^{28}$, der Suizidwillige handlungsunfähig geworden ist ${ }^{29}$ und die Erfolgsabwendung individuell zumutbar ist $\mathrm{t}^{30}$.

In der Lehre wird teilweise die weitere Meinung vertreten, dass bei einem erkennbar nicht abwägend und zielgerichtet verfolgtem Suizidgeschehen zwar keine Tötung durch Unterlassen, wohl aber die mit geringerer Strafe bedrohte Unterlassene Hilfeleistung $(\mathbb{S} 323 \mathrm{c}$ d-StGB) anzunehmen sei, wenn der Suizident nach vollendetem Versuch nur noch als Opfer seines Tuns angetroffen werde. ${ }^{31}$ Gegen eine Unterlassungsstrafbarkeit generell lässt sich einwenden, dass der freiverantwortlich gefasste Suizidwille von der Rettungspflicht entbindet. Erst wenn ein Sinneswandel des ursprünglich Suizidwilligen erkennbar wird, entsteht für den Arzt oder Angehörigen als Beschützergarant gemäss $\ 13 \mathrm{~d}-\mathrm{StGB}$ und für andere gemäss $\ 330 \mathrm{c}$ d-StGB eine Rettungspflicht. In der neueren Rechtsprechung der Oberlandes- und Landgerichte findet sich eine zunehmende Respektierung des freiverantwortlichen Suizidwillens durch Ablehnung einer Verhinderungspflicht Dritter. ${ }^{32}$ Indessen versperrt einstweilen der BGH mit seinem auch in jüngerer Vergangenheit bestätigten ${ }^{33}$ Festhalten an seiner Rechtsprechung diesen Weg.

Jenseits der in Deutschland drohenden Unterlassungsstrafbarkeit des Suizidhelfers erweist sich bereits die in Deutschland vorgenommene Vermittlung einer Schweizerischen Suizidhilfe als Beihilfe zu einem Betäubungsmitteldelikt ( $\mathbb{S} 29$ Abs. 1 lit. 6 i. V. m. 13 d-BtMG, 27 d-StGB). Denn, anders als in der Schweiz, bildet die Abgabe des todbringenden Medikaments $\mathrm{NaP}$ in Deutschland sogar mit Rezept einen strafbaren Verstoss gegen das Betäubungsmittelgesetz ( $\mathbb{S} 29$ Abs. 1 lit. 6 i. V. m. 13 d-BtMG). Obwohl die Haupttat in der Schweiz straflos bleibt, ist eine inländische Teilnahme nach deutschem Recht strafbar ( $\$ 9$ Abs. 2 Satz 2 d-StGB).

Insgesamt ergibt sich, dass die Schweiz generell gegenüber der Suizidhilfe und speziell gegenüber der organisierten aufgeschlossener als Deutschland ist. Dies drückt sich auch in den medizinischethischen Richtlinien beider Länder aus. In den Grundsätzen der deutschen Bundesärztekammer zur ärztlichen Sterbebegleitung war früher festgehalten, dass die Mitwirkung des Arztes an der Selbsttötung des Patienten dem ärztlichen Ethos widerspricht. Bis kürzlich wurde die einer pluralistischen Gesellschaft angemessenere, aber immer noch gegenüber der ärztlichen Suizidhilfe reservierte Formulierung gewählt: „Die Mitwirkung des Arztes bei der Selbsttötung ist keine ärztliche Aufgabe. "34 Dieselbe Formulierung verwandten die entsprechenden schweizerischen Richtlinien von 1995 bis 2004. ${ }^{35}$ Der deutsche Bundesärztetag 2011 hat nun beschlossen, in der Muster-Berufsordnung (MBO) für Ärzte in Bezug auf die Mitwirkung eines Arztes bei einem Suizid eines Patienten die folgende Formulierung einzufügen: „Ärztinnen und Ärzte haben Sterbenden unter Wahrung ihrer Würde und unter Achtung ihres Willens beizustehen. Es ist ihnen verboten, Patienten auf deren Verlangen zu töten. Sie dürfen keine Hilfe zur Selbsttötung leisten." Es bleibt abzuwarten, ob diese Regelung der MBO von den einzelnen Bundesländern in das ärztliche Berufsrecht übernommen und damit Rechtsverbindlichkeit erlangen wird. In den geltenden schweizerischen Richtlinien heisst es hingegen differenzierend: „Auf der einen Seite ist die Beihilfe zum Suizid nicht Teil der ärztlichen Tätigkeit, weil sie den Zielen der Medizin widerspricht. Auf der anderen Seite ist die Achtung des Patientenwillens grundlegend für die Arzt-Patienten-Beziehung. Diese Dilemmasituation erfordert eine persönliche Gewissensentscheidung 
des Arztes. Die Entscheidung, im Einzelfall Beihilfe zum Suizid zu leisten, ist als solche zu respektieren. “36 Die Entwicklung der Meinungsbildung verläuft in der Schweiz zunehmend dahin, dass die Suizidhilfe als freiwillige ärztliche Aufgabe verstanden wird, die zwar keinem Arzt aufgedrängt werden kann, aber auch aufsichts- bzw. standesrechtlich nicht ausgeschlossen erscheint, solange bei der Untersuchung, Diagnose und Abgabe die ärztlichen Sorgfaltspflichten eingehalten werden. ${ }^{37}$

Auch hinsichtlich des für die Verschreibung des todbringenden $\mathrm{NaP}$ geforderten Krankheitsbildes ist ein Meinungswandel hin zu liberaleren Positionen erkennbar. Erachtete man früher die Verschreibung nur bei einem unabwendbar zum Tode führenden Leiden als zulässig ${ }^{38}$, so genügt heute eine unheilbare, dauerhafte, schwere somatische oder sogar psychische Beeinträchtigung, die dem Patienten sein Leben auf Dauer hin nicht mehr als lebenswert erscheinen lässt. Freilich ist nach dem Bundesgericht bei rein psychischen Leiden „äusserste Zurückhaltung geboten: Es gilt zwischen dem Sterbewunsch zu unterscheiden, der Ausdruck einer therapierbaren psychischen Störung ist und nach Behandlung ruft, und jenem, der auf einem selbst bestimmten, wohlerwogenen und dauerhaften Entscheid einer urteilsfähigen Person beruht (‘Bilanzsuizid`), den es gegebenenfalls zu respektieren gilt.“"39

Die schweizerische Regierung (Bundesrat) hat 2006 eine Erweiterung der Strafbestimmung über die Suizidbeihilfe aus selbstsüchtigen Beweggründen (Art. 115 ch-StGB) ebenso abgelehnt wie die Schaffung eines Aufsichtsgesetzes zur Qualitätssicherung in der organisierten Suizidhilfe. ${ }^{40}$ Diese Entscheidung wurde zum Teil heftig kritisiert. So setzt sich die SAMW für eine Aufsicht des Bundes über die Sterbehilfeorganisationen ein. Sie erachtet es als unbefriedigend, dass gerade $\mathrm{da}$, wo es um Leben und Tod geht, nur ungenügende Sorgfaltskriterien und keine Aufsicht bestehen. ${ }^{41}$ In einer vom Bundesrat veranlassten Vernehmlassung hat sich inzwischen eine deutliche Mehrheit der Kantone, Parteien und interessierten Organisationen mit im Einzelnen kontroversen Vorschlägen für eine ausdrückliche Regelung der organisierten Suizidhilfe auf Bundesebene ausgesprochen. Am 17. 9. 2010 hat der Bundesrat eine gesetzlichen Handlungsbedarf bestimmende Botschaft zur Festlegung von Sorgfaltspflichten für Mitarbeitende von Suizidhilfeorganisationen sowie zur verstärkten Förderung der Suizidprävention und der Palliativmedizin angekündigt. ${ }^{42}$ Am 29. 6. 2011 hat Bundesrätin Sommaruga den Entscheid des Bundesrates mitgeteilt, auf eine ausdrückliche Regelung der organisierten Suizidhilfe zu verzichten, da Missbräuche bereits mit dem geltenden Recht geahndet werden könnten und eine dies konkretisierende gesetzliche Regelung Suizidhilfeorganisationen ein auch in Ärztekreisen unerwünschtes staatliches Gütesiegel verleihen würde. ${ }^{43}$

Im Spannungsfeld zwischen paternalistischem Lebensschutz und Respekt vor einem selbstbestimmten menschenwürdigen Sterben

Auf den Punkt gebracht, unterscheidet sich die rechtliche Bewertung des begleiteten Sterbens in beiden Nationen wie folgt: Kein Unterschied besteht hinsichtlich der Rechtslage für die Unterstützung eines ohnehin in Gang befindlichen Sterbevorganges. Bei dem sich zu Ende neigenden Leben ist Sterbebegleitung durch palliative Anwendung schmerzlindernder Mittel und ärztlichen Behandlungsabbruch in beiden Ländern zulässig und üblich. Hingegen bestehen Unterschiede hinsichtlich der Suizidbegleitung von Personen, die bloss sterben wollen, ohne dies alsbald zu müssen. Deutschland verbietet prinzipiell die Abgabe der zuverlässig rasch und schmerzfrei todbringenden Substanz NaP in individuell ausreichender Menge. Damit wird im Sinne der Suizidprävention dem Umstand Rechnung getragen, dass Suizidversuche häufig auf später als kurzsichtig erkannte Augenblicksentscheide in Krisensituationen zurückgehen. Mit der statistisch durchaus zutreffenden, individuell aber rein spekulativen Unterstellung, dass der Freitodversuch später bereut werde, wird ein Paternalismus praktiziert, welcher sich über das Selbstbestimmungsrecht des vollverantwortlich Suizidwilligen hinwegsetzt. Suizidwillige sind entweder zum Gebrauch unsicherer, womöglich schmerzhafter Tötungsmittel oder schicksalhaft zum Weiterleben verurteilt. Gemäss Angaben des Statistischen Bundesamtes haben sich im Jahre 2009 in Deutschland insgesamt 9.571 amtlich festgestellte Suizide ereignet. ${ }^{44}$ Mangels Verfügbarkeit eines zuverlässig schmerzfreien Tötungsmittels im Rahmen der Freitodbegleitung dürften zahlreiche dieser Freitode, und erst recht missglückte Versuche solcher, mit objektiv unnötigen Qualen verbunden gewesen sein.

In der Schweiz hingegen wird die Schleuse zu einem selbstbestimmten Freitod in Begleitung von Suizidhilfeorganisationen offen gehalten. Anders als Deutschland ist die Schweiz nicht historisch mit einer Ideologie der „Minderwertigkeit“ gewissen menschlichen Lebens und einer Praxis der Euthanasie belastet. Zudem gebietet die traditionell liberal-konservative Rechtskultur der Schweiz eher staatliche Zurückhaltung bei der Bewertung individueller Entscheidungen. Der auch im Rechtlichen anzutreffende typisch schweizerische Pragmatismus vertraut auf eine weite Ermessensspielräume sinnvoll füllende Praxis, die tunlichst nicht durch Gesetze oder Leitentscheidungen beengt werden sollte.

Grundsätzlich, wenngleich in geringerem Masse als der nördliche Nachbar, praktiziert indessen auch die Schweiz einen paternalistischen Schutz des Lebens vor unüberlegtem Freitod. ${ }^{45}$ So ist im Einzelfall zu prüfen und zu dokumentieren, dass der Entscheid, dem Leben ein Ende zu setzen, tatsächlich dem freien, wohlerwogenen und nicht bloss augenblicksbezogenen Willen des Betroffenen entspricht. Zudem kann ein Suizidwilliger das todbringende $\mathrm{NaP}$ in letaler Dosierung nicht direkt von der Suizidhilfsorganisation beziehen, sondern benötigt dafür ein ärztliches Rezept. Ein Drugstoremodell, in dem die Hilfsorganisation die Substanz „ohne weiteren Firlefanz “46 nach eigenem grosszügigem Ermessen abgeben kann, ist nach geltendem Recht nicht zulässig und hat auch rechtspolitisch keine Chance. Für die Verschreibung von $\mathrm{NaP}$ genügt der bilanzierte nachvollziehbare Suizidwunsch auf Grund einer unheilbaren schweren Beeinträchtigung. Ein unabwendbar alsbald zum Tode führendes Leiden ist dafür nicht vorausgesetzt. Wie die Ärzteschaft diese vage Begrifflichkeit handhabt, ist unbekannt. Zu vermuten steht eine uneinheitliche Praxis, in der recht viele Ärzte die Voraussetzungen weit auslegen und die Substanz im Wesentlichen bereits auf einen als ernsthaft empfundenen Suizidwunsch hin verschreiben. Dies dürfte erst recht für die sog. „Vertrauensärzte“ der organisierten Suizidhilfe gelten. So ist anzunehmen, dass jeder ernsthaft Suizidwillige letztlich einen Arzt finden wird, der zur Verschreibung von $\mathrm{NaP}$ bereit ist.

Zudem ist die Praxis der indirekten aktiven Sterbehilfe und des ärztlichen Behandlungsabbruchs in der Schweiz deutlich verbreiteter als in Deutschland. Die Intransparenz dieser Praxis begründet zumindest abstrakt die Gefahr einer unausgesprochenen individuellen Beurteilung nach der Belastbarkeit des Pflegesystems. Insofern freilich schwere Missbräuche bisher nicht bekannt wurden, profitiert die ärztliche Sterbebegleitung von dem in der Schweiz noch intakten Grundvertrauen in die Ärzteschaft. 
Nach einer umstrittenen ${ }^{47}$ Befragung findet der Autonomieanspruch im Sterben starke Zustimmung. Viele Schweizerinnen und Schweizer können sich bei unheilbarer Erkrankung vorstellen, organisierte Sterbehilfe in Anspruch zu nehmen. Viele votieren sogar für eine Erweiterung der Zulässigkeit von Sterbehilfe. Hingegen wird eine Suizidhilfe für Ausländer mehrheitlich abgelehnt. ${ }^{48}$ Die grundsätzliche Befürwortung der Möglichkeit eines assistierten Sterbens kontrastiert mit einem in der politischen Öffentlichkeit verbreiteten Misstrauen gegenüber undurchsichtiger Praktiken und dem teilweise offensiven und expansiven Auftreten der Sterbehilfeorganisationen. Dies hat die organisierte Suizidhilfe einer Art Generalverdacht ausgesetzt und hält weiterhin die Diskussion um gesetzliche Vorgaben und effiziente Kontrollmöglichkeiten wach. Die Gefahren der Überreglementierung und eines noch grösseren Sterbetourismus durch den Eindruck staatlicher Zertifizierung von „Kompetenzzentren“ zur Suizidbegleitung könnten gering wiegen im Vergleich zu dem Vorwurf mangelnder staatlicher Aufsicht über eine buchstäblich existentielle Betätigung mit evidentem Missbrauchsrisiko. Zur laufenden kontroversen Diskussion um die Inhalte eines möglichen Gesetzes und zum vergeblichen Versuch, dem im Kanton Zürich durch eine vertragliche Vereinbarung vorzugreifen, siehe den nachfolgenden Beitrag von Eicker / Fisch.

Auch wenn eine über die derzeitigen gesetzlichen Bestimmungen hinaus gehende Regelung konkret nicht in Aussicht steht, hat sich in der Schweiz das Bewusstsein der Öffentlichkeit für mögliche Missbräuche bei der organisierten Suizidbegleitung geschärft. Eine vertrauensgetragene Politik des grosszügigen Tolerierens der organisierten Sterbebegleitung ohne genaues Hinschauen wird es künftig nicht mehr geben. Das drohende Szenario eines Drugstore mit internationaler Anziehungskraft, in dem Lebensmüde ihren tödlichen Cocktail auf Wunsch gemixt erhalten, zwingt zur Aufgabe des typisch schweizerischen Pragmatismus, der mit spärlichen gesetzlichen Vorgaben auskommt und durch eine weitgehend unkontrollierte, an individueller Selbstbestimmung orientierte Praxis von Reformdruck entlastet.

\section{Fußnoten}

1 http://www.exit.ch/wDeutsch/2110002/exit_auf_einen_blick.php?navan chor=2110029, besucht am 22.02.2011.

2 Stand: 31. 12. 2009, http://www.dignitas.ch/Taetigkeitsberichte/Mitglieder_Staat_31.12.2009.

3 http://www.dignitas.ch/Taetigkeitsberichte/FTB_nach_Jahr_Domizil.pdf.

4 So die übereinstimmenden Formulierungen bei EXIT und DIGNITAS

5 Art. 14a Abs. 1 BtmG.

6 Nach dem schweizerischen Bundesgericht darf auch psychisch kranken Personen, freilich nur unter strengen Voraussetzungen, Suizidhilfe geleistet werden, vgl. BGE 133I 58, Erwägung 6.3.5.

7 Vgl. etwa „Dignitas wieder in den Schlagzeilen“, www.dignitas.ch/Weitere Texte/BBC-Interview_Stellungnahme040420009.pdf.

$8 \quad \mathrm{NZZ}$ vom 17. 4. 2001.

9 BGE 133 I 58 vom 3. 11. 2006.

$10 \mathrm{EuGH}$ 31322/07, Urteil vom 20. 1. 2011.

11 Vgl. „Mit Luftballon-Gas in den Tod“, NZZ vom 19. 3. 2008, 3.

12 Vgl. "Kein Ort zum Sterben. Nirgends", St. Galler Tagblatt vom 6. 10. 2007, 3 sowie das Interview mit L. Minelli "Sterbehilfe ist im Wohnwagen ideal”, Der Landbote vom 17. 11. 2007, 21.

13 Vgl. etwa Protokoll des Regierungsrats des Kantons Zürich vom 17. 12 2007 zu KR-Nr. 339/ 2008 und den Bericht in 20 Minuten vom 10. 10. 2008,7 .

14 Angaben nach Eidgenössisches Justiz- und Polizeidepartement EJPD: Organisierte Suizidhilfe. Vertiefte Abklärungen zu Handlungsoptionen und bedarf des Bundesgesetzgebers, http://www.bj.admin.ch/content/dam/data/ gesellschaft/gesetzgebung/sterbehilfe/ber-org-suizidhilfe-d.pdf, $8 \mathrm{ff}$.

15 http://www.dignitas.ch/WeitereTexte/dignitas_de/Jahresbericht2009.pdf, 7.

16 http://www.dignitas.ch/WeitereTexte/dignitas_de/Jahresbericht2009.pdf.

17 Zur einschlägigen schweizerischen Rechtslage generell etwa Petermann Frank Th. (o. J.) Sicherheitsfragen der Sterbehilfe, St. Gallen; Schwarzenegger Christian, in: Niggli Marcel A. / Wiprächtiger Hans (Hrsg.) Basler
Kommentar Strafrecht II, Art. 111 - 392 StGB (2010) 2. Aufl., Basel , Rn 20 vor Art. 211; Venetz Petra (2008) Suizidhilfeorganisationen und Strafrecht. Luzerner Beiträge zur Rechtswissenschaft, Zürich .

$18 \mathrm{Zu}$ letzterer Fallgruppe und ihrer rechtlichen Problematik Kunz Karl-Ludwig (2002) Sterbehilfe: Der rechtliche Rahmen und seine begrenzte Dehnbarkeit. In Donatsch A., Forster M., Schwarzenegger C. (Hrsg.): Festschrift für Stefan Trechsel zum 65. Geburtstag, Zürich, Basel, Genf, 613-630, 628 f. und $\$ 215$ II AE-Sterbehilfe.

19 Die Forderung einer entsprechenden Absicht verlagert das Problem von der objektiven auf die subjektive Tatbestandsseite und ist anzulehnen, vgl. Kunz Sterbehilfe: Der rechtliche Rahmen und seine begrenzte Dehnbarkeit. , 619. Für die Zulässigkeit der indirekten aktiven Sterbehilfe vgl. die medizinischethischen Richtlinien der Schweizerischen Akademie der Medizinischen Wissenschaften (SAMW) zur Betreuung Sterbender (http://www.samw.ch), Teil II Ziff. 1.3

20 Kunz Sterbehilfe: Der rechtliche Rahmen und seine begrenzte Dehnbarkeit, $619 \mathrm{f} .$.

21 Eser in Schönke / Schröder (2010) Strafgesetzbuch. Kommentar, 28. Auflage, München, Vorbem. $\mathbb{S} 211$ ff., Rn 23.

22 Verbreitet als „passive Sterbehilfe“ bezeichnet. Neuerdings lehnt der deutsche BGH die Differenzierung nach aktivem und passivem Handeln nach äußerlichen Kriterien ab und erweitert damit die zulässige „passive“ Sterbehilfe um Fälle des auch Komponenten aktiven Tuns umfassenden Behandlungsabbruchs, welcher durch immanente Kriterien der Behandlungsbezogenheit und der Verwirklichung des auf die Behandlung bezogenen Willens der betroffenen Person bestimmt wird, vgl. BGH 2 StR 454/09 vom 25. 6 . 2010 .

$23 \rrbracket 1901$ a I d-BGB, eingeführt durch Patientenverfügungsgesetz vom 29. 7. 2009, BGBl I 2286

24 Gemäß Art. 61 Abs. 2 ZGB, 857 OR.

25 Eser in Schönke / Schröder Strafgesetzbuch. Kommentar , Vorbem. $\mathbb{S} 211$ ff., Rn 41.

26 BGH 2, $150 \mathrm{ff}$.

27 BGH St 6, 147; 7, 272

28 BGH 13, $166 \mathrm{f}$.

29 BGH NJW 60, $1821 \mathrm{f}$.

30 BGH 7, 272; 13, 169; 32, 381

31 Dölling NJW 86; 1016; Neumann JA 87, 254 ff.

32 Nachweise bei Eser in Schönke / Schröder Strafgesetzbuch. Kommentar, Vorbem. $\int \$ 211$ ff., Rn 43.

33 BGH 32, 367 ff.

34 Grundsätze der Bundesärztekammer zur ärztlichen Sterbebegleitung vom 21. 1. 2011, Deutsches Ärzteblatt 108 (2011), A 346 f.

35 Medizinisch-ethische Richtlinien für die ärztliche Betreuung sterbender und zerebral schwerst geschädigter Patienten der Schweizerischen Akademie der Medizinischen Wissenschaften (SAMW) vom 24. 2. 1995,

Schweiz. Ärztezeitung 76 (1995), 1223 ff.

36 Medizinisch-ethische Richtlinien zur Betreuung von Patientinnen und Patienten am Lebensende vom 25. 11. 2004, Schweiz. Ärztezeitung 86 (2005), 172 ff., 174.

37 So auch BGE 133 I 58, Erwägung 6.3.4.

38 Vgl. Kunz Sterbehilfe: Der rechtliche Rahmen und seine begrenzte Dehnbarkeit. , 624

39 BGE 133 I 58, Erwägung 6.3.5.1.

40 Vgl. Bericht „Sterbehilfe und Palliativmedizin - Handlungsbedarf für den Bund?“ des EJPD vom 24. 4. 2006 sowie Ergänzungsbericht vom Juli 2007.

41 Schreiben an den Bund vom 7. 6. 2006

42 Medienmitteilung des EJPD vom 17. 9. 2010.

43 Medienmitteilung des EJPD vom 29. 6. 2011.

$44 \mathrm{http}: / /$ www.destatis.de/jetspeed/portal/cms/Sites/destatis/Internet/DE/Presse/pm/2010/10/PD10_371_232.psml.

45 Zur verfassungsrechtlichen Abwägung mit dem Selbstbestimmungsrecht Kiener Regina (2010) Organisierte Suizidhilfe zwischen Selbstbestimmungsrecht und staatlichen Schutzpflichten. Zeitschrift für Schweizerisches Recht 129, 271 - 290

46 So der Generalsekretär von DIGNITAS Minelli laut Willmann, in: DIE ZEIT Nr. 36/2001, 24.

47 Schmitt Hanspeter Ist die Schweiz mehrheitlich für die aktive Sterbehilfe? Jusletter 21. 3. 2011. Kritisiert wird der suggestive Befragungscharakter mit hochdramatischen Fallschilderungen ohne Handlungsalternativen.

48 Vgl. EXIT Info 1/2007, 4 ff.; Schwarzenegger Christian / Manzoni Patrik / Studer David / Catia Leanza (2010) Was die Schweizer Bevölkerung von Sterbehilfe und Suizidbeihilfe hält. Jusletter 13. 9. 2010 